\title{
Nanomechanics of Carbon Honeycomb Cellular Structures
}

\author{
Ziang Zhang ${ }^{\mathrm{a}}$, Alex Kutana ${ }^{\mathrm{a}}$, Yang Yang ${ }^{\mathrm{a}}$, Nina V. Krainyukova ${ }^{\mathrm{b}}$, Evgeni S. Penev ${ }^{\mathrm{a}}$, Boris I. Yakobson ${ }^{\mathrm{a}, *}$ \\ ${ }^{a}$ Department of Materials Science and NanoEngineering, Rice University, Houston, TX 77005, United States \\ ${ }^{b}$ B. Verkin Institute for Low Temperature Physics and Engineering of the National Academy of Sciences of Ukraine, 47 Lenin \\ Avenue, 61103 Kharkiv, Ukraine
}

\begin{abstract}
Recently synthesized graphitic honeycomb structures, consisting of $s p^{2}$-bonded graphene nanoribbons connected by $s p^{3}$-bonded "hinges" are investigated theoretically. Honeycombs of different "wall-chiralities" (armchair and zigzag) and sizes are studied. Simulation of the reconstruction of the hinges shows that zigzag honeycombs spontaneously rearrange, resulting in a new structure. Elastic mechanical simulations show that the Young's modulus of the structures is determined solely by the density of the hinges, regardless of the structural orientation or regularity. Compression tests display a distinct behavior of self-localized deformation, similar to that of macroscopic honeycombs. Interestingly, the failure strain of the honeycomb structure is affected significantly by its lattice size and geometrical regularity. Electronic band structures of different types of honeycombs are calculated, showing that the conductivity of armchair honeycombs follows the well-known " $3 n$ ”-dependency, while zigzag honeycombs are always metallic.
\end{abstract}

Keywords: carbon allotropes, graphitic honeycomb, 3D carbon architectures, nanomechanics

\section{Introduction}

The search for new allotropes of carbon has been an enduring effort for materials scientists [1]. Since the advent of nanoscience, this endeavor has been met with significant success leading to experimental discovery of fullerenes [2, 3], nanotubes (CNTs) [4], and graphene [5]. Atomistic simulations have provided scientists with a powerful new tool to explore

${ }^{*}$ Corresponding author.

Email addresses: kutana@rice.edu (Alex Kutana), epenev@rice.edu (Evgeni S. Penev), biy@rice.edu (Boris I. Yakobson)

Preprint submitted to Carbon potential carbon allotropes [6]. Hypothetical allotropes such as graphyne [7], carbyne [8], and many other possible candidates were proposed [9, 10, 11], with the likelihood of being experimentally realized varying by each case. With diamond and graphite being the most common carbon allotropes, a carbon allotrope combining the $s p^{3}$-bonded atoms of the former and the $s p^{2}$-bonded atoms of the latter is not only interesting, but may also possess unique properties that may give rise to potential applications $[12,13]$. Here we use the term "honeycomb" [14] to denote this type of carbon modifica- 
tion.

An early scheme for the honeycomb structure was proposed by Karfunkel and Dressler [15]. The basic geometry was constructed, and electronic structure calculations of the density of states using the modified neglect of diatomic overlap method were carried out. They concluded that this hypothetical structure had "stability comparable to that of diamond," and may exist in nature but has been overlooked. They also predicted that it may simultaneously display the properties of diamond (along the main axis) and graphite (orthogonally) [15]. Balaban et al. [13] investigated the honeycomb structure again (denoted as "diamond-graphite hybrids"), and predicted that it may be conductive along the graphitic ribbons. Bucknum et al. [16] proposed a structure (later named 'hexagonite') that shares similar features, albeit much denser than the porous honeycomb.

More recently, Park and Ihm [17] performed a computational study on the electronic structure and mechanical stability of the honeycomb. They used $a b$ initio calculations for smaller cells, and tight binding for larger cells, and discovered that the electronic structure of the honeycomb follows a similar size dependence as that of zigzag nanotubes. When the size $N$ is equal to an integer times $3 / 2$, the honeycomb is metallic, and otherwise semiconducting [17]. Regarding stability, they confirmed that the honeycomb stability is comparable to that of diamond, also consistent with previous works. A type of stable carbon foam was also proposed in [18], with structure very similar to the honeycomb, and reported to be metallic and structurally rigid.

Over the last decade, there has been a sustained activity in honeycomb research [19, 20, 21]. Kawai et al. [20] proposed a carbon architecture made up of two graphene sheets intersecting each other, which is essentially the junction ("hinge") component of the honeycomb, and Ribeiro et al. [21] studied various possible diamond-graphite hybrid structures, including the honeycomb lattice, and the transition energy barrier and pressure required for their formation from graphite. Bucknum et al. [22] studied the calculated diffraction pattern for the density-functional theory (DFT) optimized hexagonite structures and reported that the hexagonite can potentially be synthesized from cold compression of CNTs. This might also be possible for the lab fabrication of the honeycomb because of the high structural similarity of the two allotropes. Kuc and Seifert [23] conducted a density-functional based tight-binding (DFTB) study on the electronic band structure of the honeycomb lattice. They concluded that the honeycomb (called carbon foam in [23]) is mechanically stable although may become unstable against shear forces if size increases, and electronically analogous to CNTs: all armchair foams (in nanotube nomenclature) are metallic, and for zigzag structures, those with distances between junctions that are multiples of three hexagonal units are metallic as well, and otherwise semiconducting. This work also suggested 


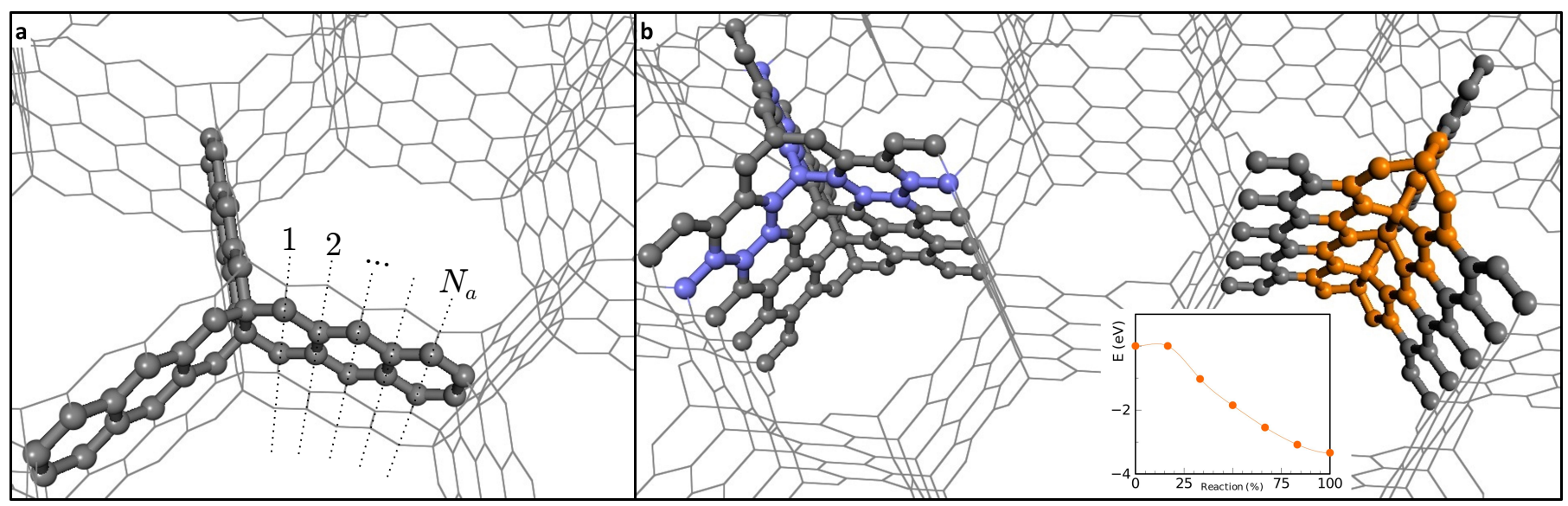

Figure 1: Structure of the honeycomb. (a) Armchair honeycomb lattice with $N_{a}=5$, using balls-and-stick representation for the unit cell. (b) Zigzag honeycomb lattice with $N_{z}=2$. Left highlight: a pristine hinge, with a zigzag unit cell marked in purple. Right highlight: a reconstructed hinge, with hinge and edge atoms marked in orange. Inset: energy per unit cell profile for the reconstruction process.

that the honeycomb structures have great potential for hydrogen storage due to their high porosity and accessibility [23]. Gas adsorption for the honeycomb has since attracted great research interest [14, 24].

Besides regular honeycomb structures with uniform pores and periodicity, several other structures of related geometry have also been proposed. Zhu et al. [25] studied the mechanical and magnetic property of a "tri-wing graphene nanoribbon" that resembles the junctions in the honeycomb, and comparable to the structure proposed by Kawai et al. [20]. Wu et al. [26] proposed a graphite structure that is essentially based on the honeycomb lattice. Despite the intensive theoretical effort, solid evidence of the experimental synthesis of the honeycomb was not presented. Very recently, Krainyukova and Zubarev [14] finally have observed exceptionally stable honeycomb lattices obtained by deposition of vacuumsublimated graphite, and demonstrated that they pos- sess high absorption level superior to that of other carbon forms such as CNTs [14]. They envisioned the honeycomb's application in both gas (hydrogen included) absorption, and structural composites [14].

\section{Computational details}

All honeycomb geometries are relaxed with DFTB [27] and the energy barrier for reconstruction of the junctions are determined by using the nudged elastic band method [28], using the DFTB+ code [29]. Electronic band structures are calculated with the VASP code [30], employing projector augmented wave pseudopotentials [31], and the localdensity approximation (LDA) to the electronic exchange and correlation. A plane wave basis with the cutoff energy of $400 \mathrm{eV}$ was used. Linear elastic properties and indentation response are obtained from empirical force-field simulations using LAMMPS [32] and the AIREBO [33] potential. 


\section{Results and discussion}

\subsection{Atomic structure and stability}

A honeycomb lattice can be viewed as multiple junctions, we refer to as "hinges", represented by a single line of carbon atoms connecting graphene nanoribbons (GNRs) running in parallel along the $z$ axis. According to the orientation of the GNRs, honeycombs can be classified into armchair and zigzag. Zigzag honeycombs can be further classified into pristine and reconstructed, depending on the configuration of the hinges. Regardless of the above mentioned classifications, all honeycomb lattices share common structural features. Because of the hexagonal lattice, the primitive cell consists of two hinges, and three GNRs. The size of the primitive cell in the $x y$-plane grows with the width of the GNRs, measured by the number of dimer or zigzag lines, $N_{a}$ or $N_{z}$, for the armchair and zigzag honeycombs, respectively. Along the $z$-axis, the lattice size remains constant regardless of the width of the GNR.

Figure 1 shows the classification of honeycombs by orientation and hinge type. Our DFT calculations show the cohesive energy of the honeycomb to be $7.56 \mathrm{eV} /$ atom, varying little with the size of the unit cell. This energy is comparable with that of graphene (7.90 eV/atom) and diamond (7.73 eV/atom), suggesting that the structure is highly stable thermodynamically. The overall thermal stability of the armchair and zigzag honeycombs has also been confirmed with constant-temperature MD simulations at $300 \mathrm{~K}$ and $1000 \mathrm{~K}$. However, a more detailed study reveals kinetic accessibility of a more stable state in zigzag honeycomb, as discussed below.

Among all the honeycombs mentioned above, the zigzag configuration is of particular interest due to its geometry apparently made up of only $s p^{2}$-bonded atoms. The first experimental report suggests that all of its atoms have $s p^{2}$ coordination and its structure is zigzag [14]. In this configuration, however, the presence of staggered dihedral geometries near the hinge atoms raises the question of how stable such all-s $p^{2}$ zigzag honeycomb structure would be. Previous work by Wu et al. [26] suggested that the zigzag lattice would converge into a lower-energy configuration with a $1 \times 1 \times 2$ reconstruction but did not explicitly state if zigzag is an unstable configuration. Here we analyze the stability of the zigzag honeycomb, and show that zigzag is a metastable state, with a very low energy barrier for reconstruction, and reconstructing even under a small perturbation. Figure $1 \mathrm{~b}$ shows the mechanism for the hinge reconstruction of zigzag honeycombs. The reconstructed structure is considerably more energetically favorable than the zigzag structure, with an energy difference of $3.34 \mathrm{eV}$ per unit cell, or $0.34 \mathrm{eV} / \AA$ of hinge length, while the transition energy barrier for this process is negligible. This means that the zigzag lattice is a local minimum, albeit a shallow one, and would reconstruct under small mechanical or thermal fluctuations. To verify our assumption we conducted a molecular dynamics simulation of zigzag lattice. The system was first optimized, and then coupled 

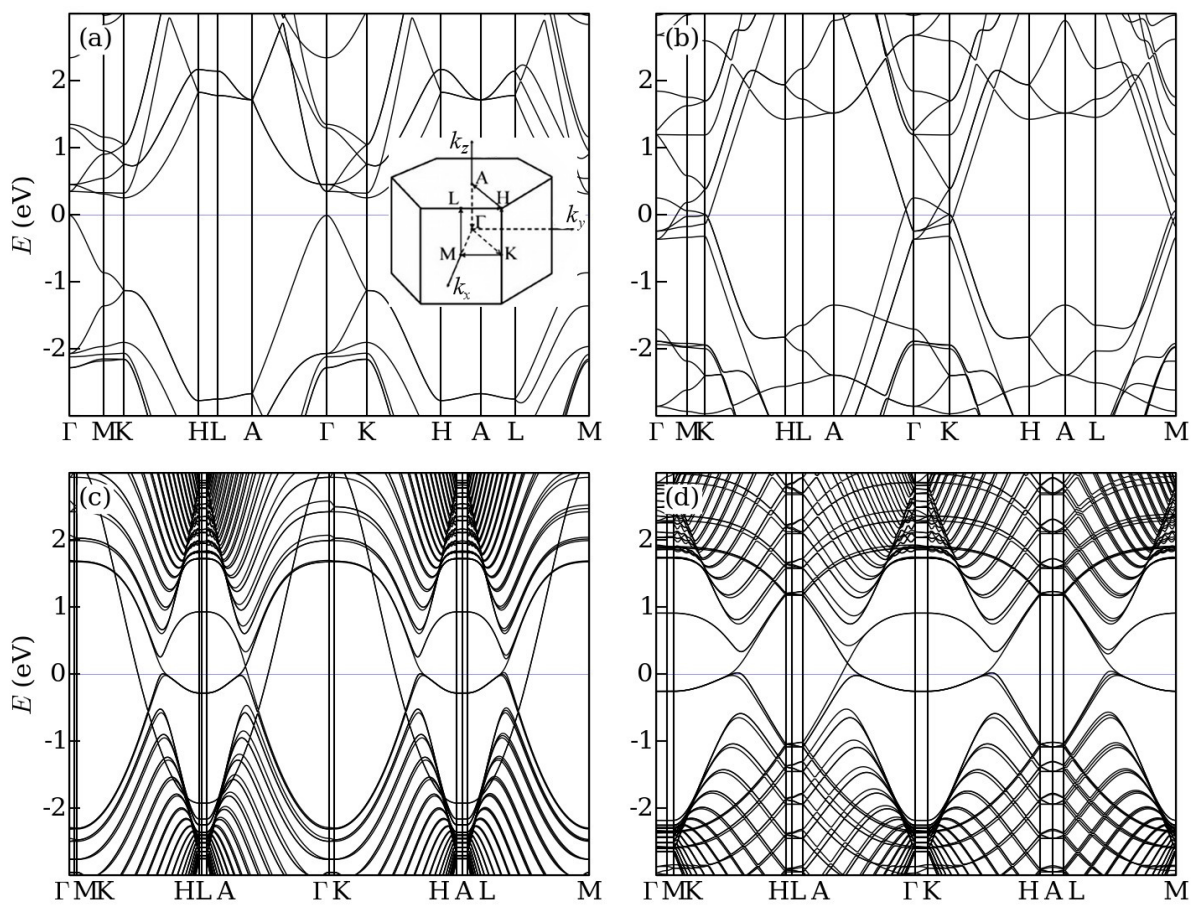

Figure 2: Electronic band structures of the honeycombs. (a) Armchair, $N_{a}=4$. (b) Armchair, $N_{a}=5$. (c) Zigzag, $N_{z}=19$. (d) Reconstructed, $N_{z}=15$. Armchair honeycombs follow the " $3 n$ " rule, whereas all zigzag honeycombs are metallic. The hinge states close to Fermi level near the A point of the BZ of zigzag honeycombs are analogous to the edge states in zigzag nanoribbons.

with a thermostat at $273 \mathrm{~K}$. We observe that, during the MD simulation, the hinge atoms irrevocably form bonds between every two atoms, effectively completing the reconstruction process.

\subsection{Basic electronic properties}

From mechanical point of view, the distinction of honeycombs into armchair and zigzag is not essential due to the elastic in-plane isotropy of graphene sheets. At the same time, electronic properties would be very sensitive to the GNR chirality. We conducted band structure calculations of armchair, zigzag, and reconstructed zigzag periodic honeycomb lattices of various sizes aiming to elucidate the effect of size and chirality. The geometries were optimized at the DFT level using the LDA functional. We clas- sify the structures using the usual convention [34] for GNRs. The armchair structure size is defined by the number $N_{a}$ of dimer lines along the ribbon, excluding the hinge atoms and it implies the relation $N_{a}=2 N-1$, where $N$ was used in Ref. [17]. We find that the band structures of armchair honeycombs follow the so-called " $3 n$ " rule, where structures with sizes $N_{a}=3 n$ and $3 n+1$ are semiconducting, and with $N_{a}=3 n+2-$ metallic, similar to armchair GNRs [34]. Previous tight binding calculations $[17,23]$ have also indicated the validity of the " $3 n$ " rule for armchair honeycombs. Here, hinge atoms are not considered as part of the ribbon when determining $N_{a}$, as they are tetrahedrally coordinated. The remaining atoms have graphene geom- 
etry corresponding to the $s p^{2}$ hybridization and contribute to the band structure that is similar to that of nanoribbons. One important difference in the electronic properties of armchair nanoribbons and honeycombs is that the honeycombs with $N_{a}=3 n+2$ appear to be truly metallic, whereas nanoribbons open a small band gap when changing the description from the nearest-neighbor tight binding to DFT [34].

A total of 20 armchair structures were calculated, with $N_{a}=1-20$. The only exception from the " $3 n$ " rule was found for the smallest structure $N_{a}=1$, which was metallic. The LDA band structures of the semiconducting $\left(N_{a}=4\right)$ and metallic $\left(N_{a}=5\right)$ armchair honeycombs are shown in Figs. $2 a$ and b. The band structures for all calculated armchair honeycombs are also shown in the supplementary Fig. S1. It is seen from Fig $2 b$ and Fig. S1 that, unlike armchair nanoribbons, the metallic behavior of honeycombs with $N_{a}=3 n+2$ is quite robust, with bands crossing the Fermi level along multiple directions in the Brillouin zone (BZ).

In contrast to the armchair structures, zigzag honeycombs of all sizes are found to be metallic, independent of whether the hinges were reconstructed or not. All the structures were nonmagnetic. The size $N_{z}$ of the zigzag honeycomb is defined by the number of the zigzag chains forming the wall, Fig. $1 b$. We have carried out geometry optimization and band structure calculations for the zigzag honeycombs with sizes $N_{z}=1-20$. The band structure of zigzag honeycomb of size $N_{z}=19$ with unreconstructed hinges is shown in Fig. 2c. Further orbital analyses show that the states near the Fermi level in the vicinity of the A point of the $\mathrm{BZ}$ are the hinge states, which are analogous to the edge states in zigzag nanoribbons. Reconstructing the hinges preserves the structure metallicity, as shown in Fig. $2 d$ for the structure of size $N_{z}=15$. The hinge states are now located around the $\Gamma$ point due to zone folding from the doubling of the lattice period in the $z$ direction. Unlike zigzag graphene ribbons which have small band gaps [34], there are several bands crossing the Fermi level in zigzag honeycombs, rendering them metallic. More band structures for zigzag and reconstructed zigzag honeycombs are shown in the supplementary Figs. S2 and S3, respectively. We note that the realistic honeycomb structures [14] are more irregular than the ones considered here, and thus have a more complicated electronic structure.

\subsection{Elastic properties}

The honeycomb possesses some of the most interesting elastic properties among carbon allotropes due to its high anisotropy. Structurally, the honeycomb lattice is densely packed along the $z$-direction, and its graphene nanoribbon walls form a large-scale honeycomb lattice also in the $x y$-plane. This system can be viewed as a nanoscale cellular solid [35], with regular hexagonal prismatic cells.

If one treats the graphene nanoribbons as "walls" of width $d$ and uniform, constant thickness $h$, in analogy to the continuum case, the ultimate characteristic of the honeycomb is its dimensionless relative den- 
(a) $d(\AA)$ $d(\AA)$

(b)

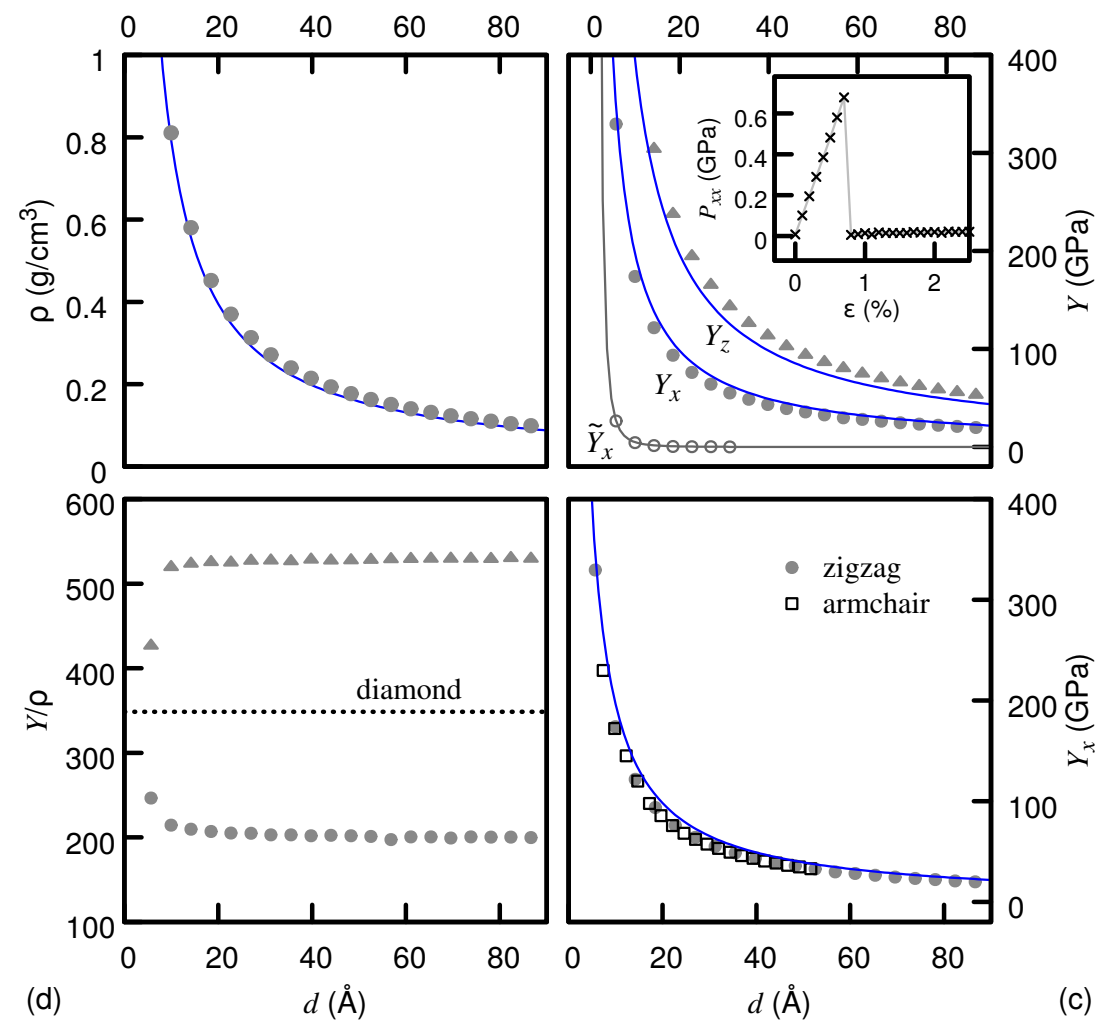

Figure 3: Elastic properties of the honeycomb. (a) Density vs. lattice size for honeycomb lattices; (b) Young's modulus of honeycombs, with both computed values (symbols) and analytic dependencies $\sim(h / d)^{m}$ (curves), with $m=1$ for $Y_{x, z}$, and $m=3$ for $\tilde{Y}_{x}$. The inset shows the $P_{x x}$ pressure as a function of strain for the honeycomb with $N_{z}=8, d \simeq 18.5 \AA$. (c) In-plane Young's modulus $Y_{x}$ for armchair and zigzag honeycombs (symbols); the full line corresponds to that in panel (b). (d) Comparison of honeycombs specific Young's moduli with that of diamond (dashed line).

sity $\bar{\rho}=\rho / \rho_{\mathrm{G}}=C h / d$, where $\rho$ is the density of the honeycomb lattice and $\rho_{\mathrm{G}}$ is that of graphene, and generally the constant $C \sim 1$. Figure 3a shows the actual $\rho$ (symbols) of all zigzag structures considered along with the corresponding $1 / d$-fit, using the standard van der Waals thickness $h=3.4 \AA$, resulting in an effective $\rho_{\mathrm{G}} \simeq 2 \mathrm{~g} / \mathrm{cm}^{3}$, with $C=2 / \sqrt{3}$ for the honeycomb lattice [35].

Within the $x y$-plane the regular-hexagonal honeycomb lattice is elastically isotropic [36] and from classical elasticity of cellular materials [35] the
Young's modulus is expected to scale as $Y / Y_{\mathrm{G}} \sim$ $(h / d)^{3}, Y_{\mathrm{G}}$ being graphene modulus. Our calculations, however, reveal a more complex behavior of the carbon honeycombs, as summarized in Fig. $3 b$. At small uniaxial compressive strain $\epsilon \lesssim 1 \%$ (cf. inset in Fig. 3b) the lattice deformation is dominated by compression of the graphene walls which can also pivot around the hinges. In this regime, as clearly seen for the example of $Y_{x}$, the in-plane modulus scales as the density, $\sim h / d$. Indeed, consider the definition $Y=\sigma(\epsilon) / \epsilon=\frac{F / A_{0}}{\Delta L / L_{0}}$, where $F$ is the elas- 


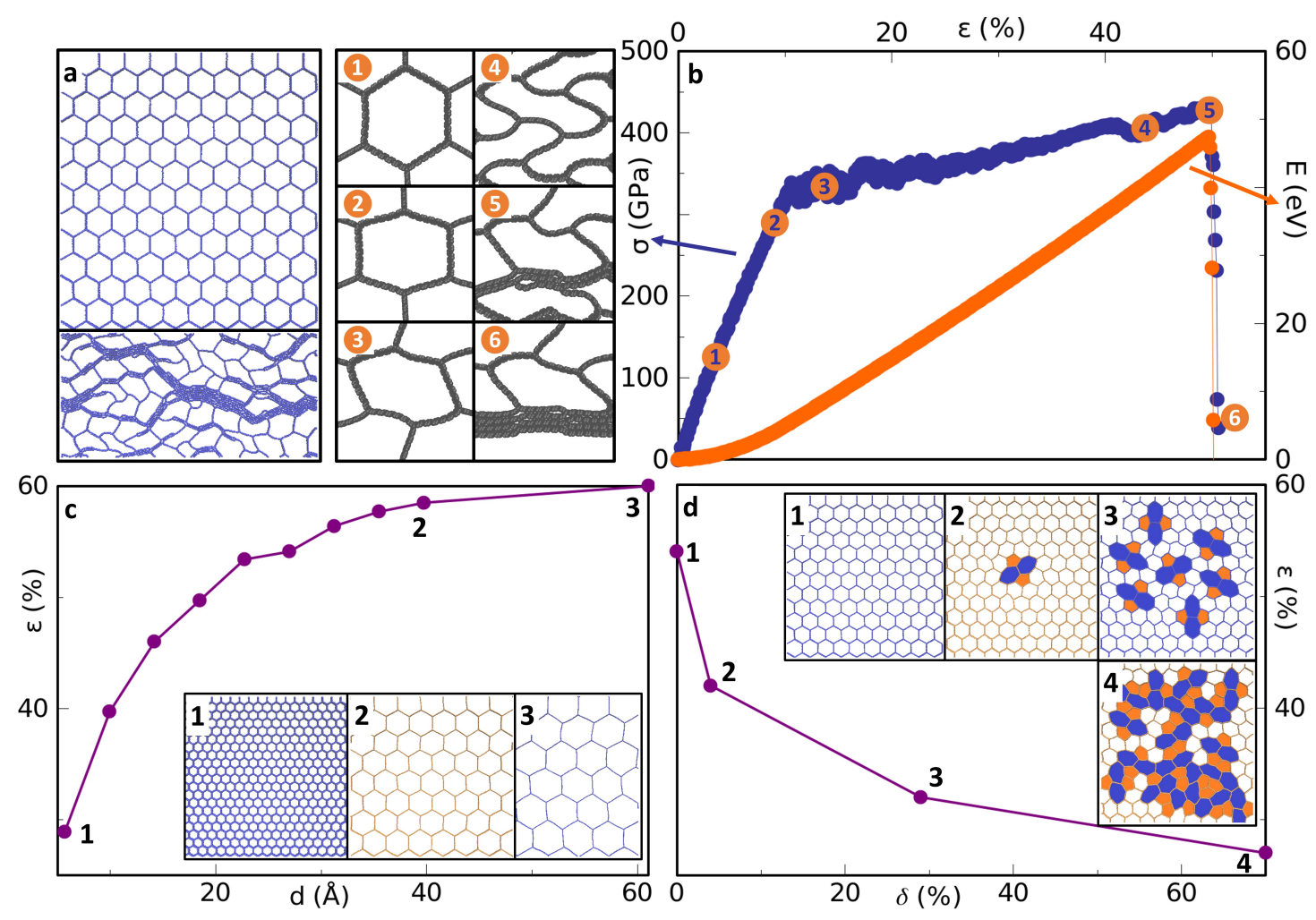

Figure 4: Compression behavior of the honeycomb. (a) Left: localized collapse of the honeycomb lattice; Right: zoomed-in view of the compression process of a honeycomb lattice, with each step numbered. (b) Stress-strain and energy-strain curves of the honeycomb during compression, with the position of each step marked. (c) Lattice size dependency of failure strain. (d) Defectdensity dependency of failure strain. In (c) and (d) the colors of the matrices are alternated for easier view and comparison.

tic response force per unit cell. Taking the zigzag honeycomb as an example, along the $x$-direction we have

$Y_{x}=\frac{F / A_{x}}{\Delta L / L_{0}}=\frac{F / A_{x}}{3 \Delta l_{x} / 3 l_{x}}=\frac{F / A_{x}}{f / a_{x}} Y_{\mathrm{G}}=\frac{F / \sqrt{3} l_{x} l_{z}}{f / h l_{z}} Y_{\mathrm{G}}$

Here, $l_{x}=d$ is the width of the constituent GNR in $x$-direction, $l_{z}$ is the length of the GNR wall in $z$ direction, $f$ is the response force of the GNR. In this case, we have $F=f$, and therefore

$$
Y_{x} / Y_{\mathrm{G}}=\frac{1}{\sqrt{3}} \frac{h}{d}
$$

For a quantitative estimate, setting $Y_{\mathrm{G}}=1 \mathrm{TPa}$, we get

$$
Y_{x}(d)=\frac{1.96 \AA}{d} \mathrm{TPa} .
$$

This analytic expression is plotted in Fig. 3b and appears to describe well the actually calculated $Y_{x}$. For larger $\epsilon$, the graphene walls bend and we recover the familiar scaling [35], $\tilde{Y} / Y_{\mathrm{G}} \sim(h / d)^{3}$, where the tilde is used merely to distinguish the modulus characteristic for this regime.

Similarly, we deduce the Young's modulus of the honeycomb in $z$-direction (note that here for an orthorhombic non primitive unit cell we have $F=6 f$ ):

$$
Y_{z} / Y_{\mathrm{G}}=\frac{F / A_{z}}{f / a_{z}}=\frac{F / 3 \sqrt{3} d^{2}}{f / h d}=\frac{2}{\sqrt{3}} \frac{h}{d} .
$$


From Eqs. (2) and (4) it follows that the in-plane/outof-plane anisotropy of the honeycomb modulus is $Y_{x} / Y_{z}=1 / 2$.

We have also found that the Young's moduli of the honeycomb structure is completely insensitive to the lattice orientation of the GNRs, as the armchair and zigzag honeycombs show overlapping trend lines of Young's moduli vs. lattice size as shown in Fig. 3c. Interestingly, elastic simulations on reconstructed honeycombs show exactly the same values of Young's moduli as those of zigzags.

The results discussed above suggest that the elastic strength of the honeycomb is only affected by the density of hinges. This may be of convenience to gauge or tune the elastic mechanical properties of experimentally obtained honeycombs, as hinge density is the only relevant parameter. We note also that the modulus of the honeycomb lattice per unit density (specific modulus) is comparable to, and specifically in z-direction, is higher than that of diamond, Fig. 3d. This could possibly mean that, with larger $d$, the honeycomb is one of the strongest foam-like material ever synthesized.

\subsection{Nanomechanical nonlinear response}

\subsubsection{Uniaxial compression}

Another important mechanical aspect of graphene honeycombs lies in their nonlinear response. The interesting phenomenon of localized compression was observed in macroscopic "honeycombs" made of stacked straws [37]. Albeit microscopic, the honeycomb structure is similar to the macroscopic "honey- combs" regarding its highly organized porosity. With this apparent structural similarity, would the honeycomb also exhibit the self-localized deformation upon compression? Furthermore, if this localization exists, would the yield strain be affected by the lattice size and/or defects in the honeycomb? To answer these questions, we conduct MD simulations of uniaxial compression on bulk honeycomb in the direction perpendicular to the hinges.

After initial optimization, the simulation box is reduced along the $y$-axis in 700 steps to simulate uniaxial compression. In each compression step, the lattice size is reduced by a constant length equal to $0.1 \%$ of the original cell size $L_{y}$, and fully relaxed along the two other axes. Both geometrical optimization and thermal movements are performed in each step. By the end of the simulation, the box is compressed by $70 \%$.

Our first set of simulations shows that, except for the smallest lattices, self-localized compression occurs for all honeycombs, regardless of lattice size or size of simulation box. The lattice first compress uniformly with walls of each cell bent, until at a critical strain level, the walls of one lattice touch and adhere, forming a nucleation point for the subsequent collapse of a line of cells, while the other lattices outside of the band remain intact, hence "localized compression", Fig. 4a. The inter-wall adhesion occurs via van der Waals interactions, without any rearrangements of covalent bonds. If further compression is applied, the system may show several more steps of 
localized compression, until completely collapsed. Each localized compression step is irreversible. We have calculated the DFT-LDA band structure of a collapsed honeycomb and compared it with the one of the original system. The band structure of the collapsed zigzag honeycomb of size $N_{z}=6$ is shown in Fig. S4. It is seen that the band structure is modified; however, the system remains metallic and the main features of electronic bands are preserved. This is not very surprising as the topology (connectivity) of the system does not change as a result of the collapse. No new bonds are formed, and interatomic distances do not change significantly, as collapse occurs chiefly through changing the bond angles. Thus, in the nearest-neighbor tight binding picture the electronic structure of the system will stay nearly same. In the actual DFT calculation, the changes of the band structure will be more significant, but drastic changes are not expected, as also evidenced by Fig. S4.

We next look into the dependency of failure strain $\epsilon_{\mathrm{cr}}$ on the lattice size. The systems simulated contain unit cells varying from $N_{z}=4$ to $N_{z}=62$. The size of the simulation boxes is set as close to $50 \times 50 \mathrm{~nm}^{2}$ as possible, by repeating the unit cells in $x$ - and $y$-directions, although some minor variations are inevitable. Since our preliminary simulations show that the failure strain is not sensitive to the size of the simulation box, we can be assured that the difference we observe between systems results entirely from different lattice sizes, rather than different simulation box sizes. Our results show that the buckling strain is $\sim 10 \%$ for systems of different sizes, Fig. 4b. Interestingly, this phenomenon can be explained by the Euler buckling theory. For a beam of length $d$, moment of inertia $I$, and Young's modulus $Y$, we have the critical load for buckling as $P_{\text {cr }}=n^{2} \pi^{2} Y I / d^{2}$. Here $n$ is the end constraint factor for beams, with $n=1$ for both ends hinged, and $n=2$ for both ends fixed. It can be deducted that for honeycomb structures of regular hexagons, regardless of the wall size $d$, the critical strain for buckling is always $10 \%$ [35]. This is confirmed by our simulation results of different lattice sizes. Figure $4 \mathrm{~b}$ shows the result for the $N_{z}=26$ structure. At the same time, the failure strain of the honeycomb increases with the lattice size, approaching about $60 \%$ for large lattice sizes, as seen in Fig. 4c.

Next, we conduct a second set of simulations to analyze the effect of defects on the localized compression behavior, Fig. 4d. For all the systems in this group, the simulation box is about $48 \times 50 \mathrm{~nm}^{2}$ in the $x y$-plane, and one unit cell in z-direction, and contains 8880 atoms. Four different honeycomb systems with different defect concentrations are studied. The first was defect free, the second contains a single Stone-Wales (SW) defect in the whole box, the third one contains $30 \%$ defective cells, and the fourth has $75 \%$ defective cells. MD simulations shows that the energy of a single SW defect is $14.0 \mathrm{eV}$. Considering that a SW defect involves relocation of 122 atoms, this energy penalty is minimal and is not expected to 


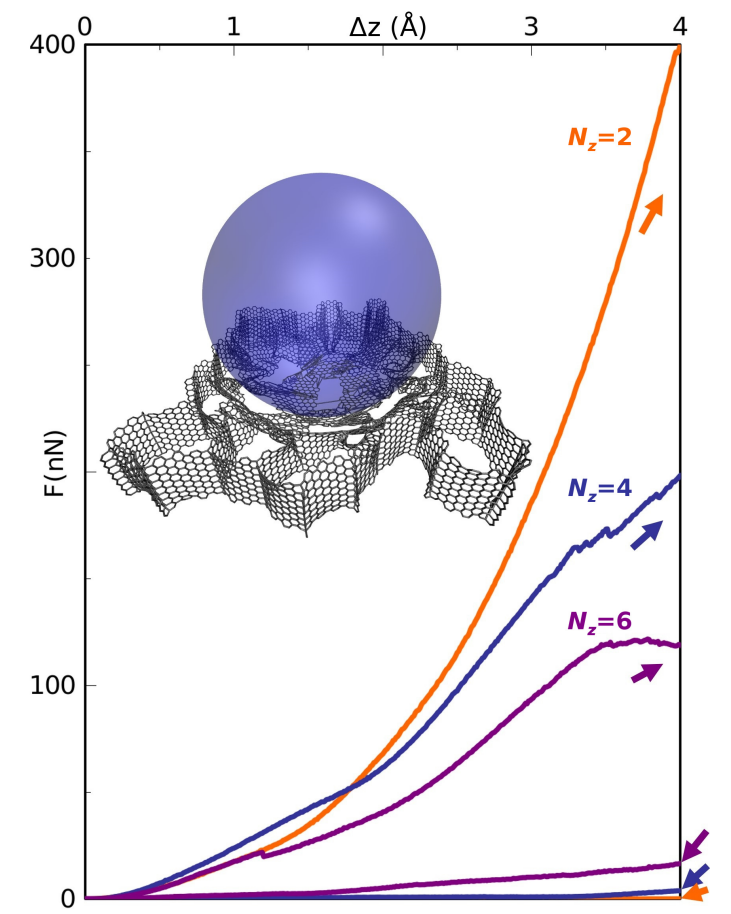

Figure 5: Simulated indentation (force $F$ vs. depth $\Delta z$ ) of the zigzag honeycomb along $z$-direction for different sizes $d$. Arrows on indicate the direction of $\Delta z$ change. The inset shows the indenter-honeycomb for the largest $d$ at maximum compression/loading depth.

significantly affect the stability of the system.

Based on the above compression tests, one can conclude that i) microscopic honeycombs do exhibit self-localized compression behavior, similar to its macroscopic counterpart; ii) the critical strain of the localized compression is increases the lattice size $d$, approaching $\simeq 60 \%$ for the largest lattices; iii) defects have a strong effect on the toughness of the honeycomb, with more defects leading to easier failure under uniaxial compression by providing nucleation sites for localized compression to initiate. The last finding would be significant for realistic structures with defects, where, compared to the perfect case, one could expect a nearly twofold reduction in the failure strain.

\subsubsection{Indentation test of the honeycomb lattice}

In order to probe the non-linear response of the honeycomb along the $z$-direction, we perform MD indentation tests. This setup corresponds closely to typical experimental tests. In each test, a finite-size honeycomb slab of dimensions $120 \times 120 \times 20 \AA^{3}$ is placed in a vacuum simulation box of $300 \times 300 \times$ $300 \AA^{3}$. The test is repeated three times for honeycombs of different lattice sizes $N_{z}$. After relaxation and thermal equilibration, a spherical indenter with a diameter of $60 \AA$ is pressed down onto the slab until reaching the vertical displacement $\Delta z$ of $15 \AA$. The indentation process is slow enough to allow the system sufficient time steps to thermally equilibrate. After reaching the vertical limit of $15 \AA$, the system is unloaded by moving the indenter up and the slab may retain some deformation (if plastic deformation has occurred) or recover to the original shape elastically. The procedure is similar to one used in our earlier work [38].

From the result of our indentation tests, we see that, honeycomb lattices of all three tested sizes $\left(N_{z}=2,4\right.$, and 6) cannot restore elastically after indentation, resulting in hysteresis in the load vs. indentation depth curves, Fig. 5. During the indentation, the structures undergo significant plastic deformations realized through rearrangements of covalent bonds. Such indentations could thus potentially be useful for creation of covalent interlayer 
links $[38,39,40]$. We also find that similar to linear elasticity, honeycombs with smaller lattice sizes have stronger mechanical response against indentation.

\section{Conclusion}

In this work, we studied the structural variation, electronic band structure, elastic properties, and localized compression of the graphitic honeycomb. Structurally, we classify honeycombs into three types according to their lattice orientation: armchair, zigzag, and reconstructed, among which zigzag and reconstructed share the GNR orientation, and armchair and reconstructed share the same hinge geometry. The stability of zigzag honeycombs is investigated, and we find that the zigzag is a metastable state that would immediately transform into the reconstructed honeycomb, as confirmed subsequently by MD simulations. The electronic band structure calculations show that armchair honeycombs do follow a periodic law analogous to armchair GNRs or zigzag CNTs, as reported previously. It also reveals that hinges play a bigger role than GNRs for the electronic structure of reconstructed honeycombs. The linear elastic mechanical tests reveal that the elastic strength of the honeycomb is dominated by the hinge density and is unaffected by the types of hinges and GNRs. Finally, we observe a highly localized compression behavior of the honeycomb, the yield strain of which is largely affected by the lattice size and the defects which provide nucleation sites for failure initiation.

\section{Acknowledgments}

This work was funded by U.S. Air Force Office of Scientific Research Grant No. FA9550-13-1-0151. Computer resources were provided by the DAVinCI cluster at Rice University acquired with funds from NSF grant OCI-0959097. This research also used resources provided by the ERDC DoD Supercomputing Resource Center.

\section{References}

[1] A. Hirsch, The era of carbon allotropes, Nat. Mater. 9 (2010) 868-871.

[2] W. Kratschmer, L. D. Lamb, K. Fostiropoulos, D. R. Huffman, Solid $c_{60}$ : a new form of carbon, Nature 347 (1990) 27.

[3] H. W. Kroto, J. R. Heath, S. C. O'Brien, R. F. Curl, R. E. Smalley, $\mathrm{C}_{60}$ : buckminsterfullerene, Nature 318 (1985) $162-163$.

[4] S. Iijima, Helical microtubules of graphitic carbon, Nature 354 (1991) 56-58.

[5] K. S. Novoselov, A. K. Geim, S. V. Morozov, D. Jiang, Y. Zhang, S. V. Dubonos, I. V. Grigorieva, A. A. Firsov, Electric field effect in atomically thin carbon films, Science 306 (2004) 666-669.

[6] F. Diederich, Carbon scaffolding: building acetylenic allcarbon and carbon-rich compounds, Nature (1994) 199207. 
[7] S. W. Cranford, M. J. Buehler, Mechanical properties of graphyne, Carbon 49 (2011) 4111-4121.

[8] M. Liu, V. I. Artyukhov, H. Lee, F. Xu, B. I. Yakobson, Carbyne from first principles: chain of $\mathrm{C}$ atoms, a nanorod or a nanorope, ACS Nano 7 (2013) 1007510082 .

[9] V. Varshney, S. S. Patnaik, A. K. Roy, G. Froudakis, B. L. Farmer, Modeling of thermal transport in pillaredgraphene architectures, ACS Nano 4 (2010) 1153-1161. doi:10.1021/nn901341r.

[10] A. K. Singh, J. Lu, R. S. Aga, B. I. Yakobson, Hydrogen storage capacity of carbon-foams: Grand canonical Monte Carlo simulations, J. Phys. Chem. C 115 (2011) 2476-2482. doi:10.1021/jp104889a.

[11] V. Georgakilas, J. A. Perman, J. Tucek, R. Zboril, Broad family of carbon nanoallotropes: classification, chemistry, and applications of fullerenes, carbon dots, nanotubes, graphene, nanodiamonds, and combined superstructures, Chem. Rev. 115 (2015) 4744-4822.

[12] J. Fayos, Possible 3D carbon structures as progressive intermediates in graphite to diamond phase transition, J. Solid State Chem. 148 (1999) 278-285.

[13] A. T. Balaban, D. J. Klein, C. A. Folden, Diamondgraphite hybrids, Chem. Phys. Lett. 217 (1994) 266-270.

[14] N. V. Krainyukova, E. N. Zubarev, Carbon honeycomb high capacity storage for gaseous and liquid species, Phys. Rev. Lett. 116 (2016) 055501.

[15] H. R. Karfunkel, T. Dressler, New hypothetical carbon allotropes of remarkable stability estimated by MNDO solid-state SCF computations, J. Am. Chem. Soc. 114 (1992) 2285-2288.

[16] M. J. Bucknum, R. Hoffmann, A hypothetical dense 3, 4connected carbon net and related $\mathrm{B}_{2} \mathrm{C}$ and $\mathrm{CN}_{2}$ nets built from 1, 4-cyclohexadienoid units, J. Am. Chem. Soc. 116 (1994) 11456-11464.

[17] N. Park, J. Ihm, Electronic structure and mechanical stability of the graphitic honeycomb lattice, Phys. Rev. B 62 (2000) 7614.
[18] K. Umemoto, S. Saito, S. Berber, D. Tománek, Carbon foam: Spanning the phase space between graphite and diamond, Phys. Rev. B 64 (2001) 193409.

[19] M. Bucknum, I. Stamatin, E. Castro, A chemically intuitive proposal for the structure of n-diamond, Mol. Phys. 103 (2005) 2707-2715.

[20] T. Kawai, S. Okada, Y. Miyamoto, A. Oshiyama, Carbon three-dimensional architecture formed by intersectional collision of graphene patches, Phys. Rev. B 72 (2005) 035428.

[21] F. J. Ribeiro, P. Tangney, S. G. Louie, M. L. Cohen, Structural and electronic properties of carbon in hybrid diamond-graphite structures, Phys. Rev. B 72 (2005) 214109.

[22] M. J. Bucknum, E. A. Castro, The carbon allotrope hexagonite and its potential synthesis from cold compression of carbon nanotubes, J. Chem. Theory Comput. 2 (2006) $775-781$.

[23] A. Kuc, G. Seifert, Hexagon-preserving carbon foams: Properties of hypothetical carbon allotropes, Phys. Rev. B 74 (2006) 214104.

[24] N. Park, S. Hong, G. Kim, S.-H. Jhi, Computational study of hydrogen storage characteristics of covalent-bonded graphenes, J. Am. Chem. Soc. 129 (2007) 8999-9003.

[25] L. Zhu, J. Wang, T. Zhang, L. Ma, C. W. Lim, F. Ding, X. C. Zeng, Mechanically robust tri-wing graphene nanoribbons with tunable electronic and magnetic properties, Nano Lett. 10 (2010) 494-498.

[26] M. Wu, X. Wu, Y. Pei, Y. Wang, X. C. Zeng, Threedimensional network model of carbon containing only $s p^{2}$-carbon bonds and boron nitride analogues, Chem. Commun. 47 (15) (2011) 4406-4408.

[27] M. Elstner, D. Porezag, G. Jungnickel, J. Elsner, M. Haugk, T. Frauenheim, S. Suhai, G. Seifert, Self-consistent-charge density-functional tight-binding method for simulations of complex materials properties, Phys. Rev. B 58 (1998) 7260.

[28] G. Henkelman, B. P. Uberuaga, H. Jónsson, A climb- 
ing image nudged elastic band method for finding saddle points and minimum energy paths, J. Chem. Phys. 113 (2000) 9901-9904.

[29] B. Aradi, B. Hourahine, T. Frauenheim, DFTB+, a sparse matrix-based implementation of the DFTB method, J. Phys. Chem. A 111 (2007) 5678-5684.

[30] G. Kresse, J. Furthmüller, Efficiency of ab-initio total energy calculations for metals and semiconductors using a plane-wave basis set, Comput. Mater. Sci. 6 (1996) 1550.

[31] P. E. Blöchl, Projector augmented-wave method, Phys. Rev. B 50 (1994) 17953.

[32] S. Plimpton, Fast parallel algorithms for short-range molecular dynamics, J. Comput. Phys. 117 (1995) 1-19.

[33] S. J. Stuart, A. B. Tutein, J. A. Harrison, A reactive potential for hydrocarbons with intermolecular interactions, J. Chem. Phys. 112 (2000) 6472-6486.

[34] Y.-W. Son, M. L. Cohen, S. G. Louie, Energy gaps in graphene nanoribbons, Phys. Rev. Lett. 97 (21) (2006) 216803.

[35] L. J. Gibson, M. F. Ashby, Cellular Solids: Structure and Properties, Cambridge Solid State Science Series, Cambridge University Press, 1999.

[36] L. D. Landau, E. M. Lifshitz, Theory of elasticity, Vol. VII of Course of theoretical physics, Pergamon Press, Oxford, 1986.

[37] S. Papka, S. Kyriakides, Biaxial crushing of honeycombs:-Part 1: Experiments, Int. J. Solids Struct. 36 (1999) 4367-4396.

[38] S. Ozden, Y. Yang, C. S. Tiwary, S. Bhowmick, S. Asif, E. S. Penev, B. I. Yakobson, P. M. Ajayan, Indentation tests reveal geometry-regulated stiffening of nanotube junctions, Nano Lett. 16 (2016) 232-236.

[39] T. Trevethan, P. Dyulgerova, C. D. Latham, M. I. Heggie, C. R. Seabourne, A. J. Scott, P. R. Briddon, M. J. Rayson, Extended interplanar linking in graphite formed from vacancy aggregates, Phys. Rev. Lett. 111 (2013) 095501. doi:10.1103/PhysRevLett.111.095501.
[40] R. P. Hardikar, A. Samanta, A. Manjanath, A. K. Singh, Vacancy mediated clipping of multilayered graphene: A precursor for 1, 2 and $3 \mathrm{~d}$ carbon structures, Carbon 94 (2015) 67 - 72 . doi:http://dx.doi.org/10.1016/j.carbon.2015.06.040. 


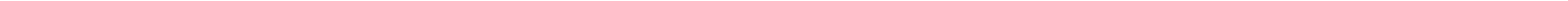

